\section{Stimulierung der Atmung durch Bradykinin und Kallidin}

Die nach der Methode von KonzeTT und RössLER ${ }^{1}$ registrierbare bronchokonstriktorische Wirkung des Bradykinins am Meerschweinchen (COLliER et al. ${ }^{2}$, BOISSONNas et al. ${ }^{3}$, KonzeTT und Stürmer ${ }^{4}$ ) lässt sich - im Gegensatz zu dem durch Histamin verursachten Bronchospasmus - durch Salicylate, z.B. Aspirin, verhindern (Collier et al. ${ }^{2} b$ ).

(1) Am spontan atmenden Meerschweinchen und Kaninchen in Urethannarkose verursachten kleine Bradykinindosen $(1-8 \mu \mathrm{g} / \mathrm{kg}$ i.v.), die offensichtlich noch nicht bronchokonstriktorisch wirkten, eine starke Hyper- und Tachypnoe, wobei Atemfrequenz und Atemvolumen auf das Doppelte bis Dreifache des Ausgangswertes anstiegen. Diese Wirkung setzte wenige Sekunden nach der Injektion ein und hielt 4-8 min lang an. Hohe Bradykinindosen (20-40 $\mu \mathrm{g} / \mathrm{kg}$ i.v.) führten an Meerschweinchen zu einer 10-30 sec dauernden Apnoe.

Ein uns von Herrn Professor Werle, München, zur Verfügung gestelltes hochgereinigtes Kallidinpräparat (aus Inkubaten von Rinderserum mit SubmaxillarisKallikrein; 1 Einheit entsprach der Wirksamkeit von $1 \mathrm{~kg}$

Beeinflussung der tachypnoischen Wirkung von Bradykinin, Kallidin, Histamin und Veratrin durch Vagotomic, Aludrin und Aspirin. Ergebnisse von Versuchen an 14 .Mecrschweinchen (100-700 g) in Urethannarkose $(1,3-1,5 \mathrm{~g} / \mathrm{kg}$ s.c. $)$. - Bradykinin $(1-8 \mu \mathrm{g} / \mathrm{kg} \mathrm{i} . \mathrm{v}$.$) :$ Kallidin $(2-6 \mathrm{E} / \mathrm{kg}$ i.v. $)$; Histamin $\left(2-20 \mu \mathrm{g} / \mathrm{kg}_{\mathrm{g}}\right.$ i.v. $)$; Veratrin $(5-20 \mu \mathrm{g} / \mathrm{kg}$ i.v.); Aludrin (Isoproterenol, 8-16 $\mu \mathrm{g} / \mathrm{kgr}$ i.v. $111 \mathrm{sec} v \mathrm{vor}-$ her); Aspirin (A eetylsalicylsaure, $4-40 \mathrm{mg} / \mathrm{kg} \mathrm{i} . \mathrm{s} 5 \mathrm{~min}$ vorher).

\begin{tabular}{|c|c|c|c|}
\hline & \multicolumn{3}{|c|}{ Tachypnoc } \\
\hline & $\begin{array}{l}\text { Bradykinin } \\
\text { hallidin }\end{array}$ & Histamin & Veratrin \\
\hline Vagotomie & aufgehoben & aufgehoben & aufgehoben \\
\hline Aludrin & unberinflusst & aufgehoben & unberinflusit \\
\hline Aspirin & aufgehoben & unbeeinflusst & unberinflust \\
\hline
\end{tabular}

\section{Identifizierung von Substanzen durch Simultan-Chromatographie isotopenmarkierter Verbindungen ${ }^{1}$}

Die inzwischen relativ niedrigen Preise für ${ }^{14} \mathrm{C}$-Präparate sowie geeignete autoradiographische Methoden rechtfertigen heute zur Sicherung von Untersuchungsergebnissen eine weitgehende Radiokontrolle auch dort, wo die Einrichtungen eines Isotopenlabors nicht vorhanden sind ${ }^{2}$.

Die Schwierigkeiten bei der Identifizierung unbekannter Naturstoffe auf dem Papierchromatogramm sind bekannt. Das bisher gebräuchlichste Verfahren, durch parallel laufende Testgemische die einzelnen Komponenten eines unbekannten Gemisches zu lokalisieren, scheitert in vielen Fällen an der Retention oder Sekundäradsorption einzelner Flecke und an farbverändernden lokalen Reaktionen auf dem Papier, beides I'hänomene, die durch begleitende Ballaststoffe erzeugt werden und beim Testgemisch nicht gegeben sind. synthetischem Bradykinin am isolierten Rattenuterus) stimulierte in vergleichbarer I) (osierung $(2-6 \mathrm{E} / \mathrm{kg}$ i.v.) ebenfalls die Atmung, indem es an Meerschweinchen und Kaninchen zu Hyper- und Tachypnoe führte und das Atemvolumen erhöhte.

Nach bilateraler Vagotomic liess sich die atmungsstimulierende Wirkung des Bradykinins und Kallidins nicht mehr aluslösen. Salicylate (4-40 $\mathrm{mg} / \mathrm{kg}$ Aspirin i.v.) verhinderten sie vollständig (Tabelle).

(2) Auch kleine I) osen levatrin $(5-20 \mu \mathrm{g} / \mathrm{kg}$ i.v.) wirkten am Meerschweinchen tachypnoisch. Die Veratrinwirkung liess sich ebenfalls durch Vagotomie, nicht jedoch durch Salicylate verhindern (Tabelle).

(3) I)ie tachypnoische Wirkung des Histamins (2-20 $\mu \mathrm{g} /$ $\mathrm{kg}$ ) ist mit einer bronchokonstriktorischen Wirkung verknüpft. Im Gegensaty zur Bradykinin-, Nallidin- und Veratrinwirkung wird sie durch Broncholytica (Aludrin) aufgehoben (Tabelle).

Die ausführliche Veröffentlichung erscheint in Naunym Schmiedeberg's Irch. exp. I'ath. I'harmak.

Summary. In guinea pigs and rabbits small doses of bradykinin and kallidin (1-8 $\mu \mathrm{g} / \mathrm{kg}$ i.v.), which have no bronchoconstrictor effect, stimulate respiration causing tachy-and hyperpnea. Bilateral vagotomy as well as salicylates (4-40 $\mathrm{mg} / \mathrm{kg}$ i.v.) abolish these respiratory responses.

V. (iJURIŚ, H. HElckE,

J. HoLTz und E. WESTERMANN

Pharmakologisches Institut der l'niversität Frankfurt a. M. (Deutschland), 30. April 1962.

1 H, Konzetr und R. Rössler, Arch. exp. l'ath. I'hammak, 10:5, $71(1 ! 10)$.

2 (a) H. (). J. Colder, J. A. Holciate, H. SCHACHTER und I'. (a. Snordey, J. Physiol. 149, 51 P' (1959). - (b) l3rit. J. Pharmacol. 1.; 2900 (1916it).

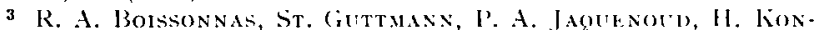

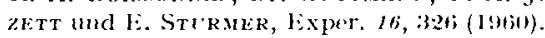

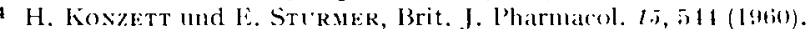

Durch Verwendung ${ }^{14} \mathrm{C}$-markierter Testsubstanzen, deren Nenge unter der chemischen Nachweisgrenze liegt, deren spezifische . Iktivitä jedoch zur Autoradjographic des Chromatogramms ausreicht (für Aminosäuren $z .13$. $5-10 \mathrm{mC} / \mathrm{m} . \mathrm{l}$ ) werden für das Testgemisch absolut, gleiche Bedingungen geschaffen. Simultan auf gleichem Startpunkt aufgetragen unterliegt dieses auch während der Chromatographie den gleichen physiko-chemischen Bedingungen wie das Untersuchungsgemisch, obwohl es später getrennt von diesem erfasst werden kann. Jeim Vergleich des angefärbten Chromatogramms mit seinem Autoradiogramm schliessen geringste Abweichungen von Form oder latge der lilecken eine Identität bereits aus.

I Fur die finanzielle [nterstutzung dieser Arbeiten danken wa den Bundesministerum fur Atomenergie und Wasserwirtschaft.

a 1)urch Verwendung empfindlicher Röntgenpapiere kann be relatis kurzem kontakthelichten (einiore Swndent) ein eindeutios Erorebnis mit gerungsten - weit unter der Freigrenze liengenden - Aktivitaten erzielt werden. 